\title{
HÖLDER'S INEQUALITY: SOME RECENT AND UNEXPECTED APPLICATIONS
}

\author{
N. ALBUQUERQUE, G. ARAÚJO, D. PELlEGRINO, AND J.B. SEOANE-SEPÚlVEDA
}

\begin{abstract}
Hölder's inequality, since its appearance in 1888, has played a fundamental role in Mathematical Analysis and it is, without any doubt, one of the milestones in Mathematics. It may seem strange that, nowadays, it keeps resurfacing and bringing new insights to the mathematical community. In this expository article we show how a variant of Hölder's inequality (although wellknown in PDEs) was essentially overlooked in Functional Analysis and has had a crucial (and in some sense unexpected) influence in very recent and major breakthroughs in Mathematics. Some of these recent advances appeared in 2012-2014 and include the theory of Dirichlet series, the famous Bohr radius problem, certain classical inequalities (such as Bohnenblust-Hille or HardyLittlewood), or even Mathematical Physics.
\end{abstract}

\section{Contents}

1. Introduction

2. Motivation: some interpolative puzzles

3. Hölder's inequality revisited

4. Some useful inequalities

4.1. Khinchine's inequality

4.2. Kahane-Salem-Zygmund's inequality

4.3. A corollary to Minkowski's inequality

5. Recent "unexpected" applications to classical problems

5.1. The Bohnenblust-Hille inequality with subpolynomial constants 12

5.2. Quantum Information Theory 16

5.3. Power series and the Bohr radius problem 17

5.4. Hardy-Littlewood's inequality constants 20

5.5. Separately summing operators 21

References

\section{Introduction}

When Leonard James Rogers (1862-1933) and Otto Hölder (1859-1937) discovered, independently, the famous inequality that (nowadays) holds Hölder's name

2010 Mathematics Subject Classification. 46B70, 46G25, 47A63, 47L22, 47H60, 30B50.

Key words and phrases. Hölder's inequality, Minkowki's inequality, interpolation, Bohr radius, Quantum Information Theory, Hardy-Littlewood's inequality, Bohnenblust-Hille's inequality, Khinchine's inequality, Kahane-Salem-Zygmund's inequality, absolutely summing operators.

D. Pellegrino and J.B. Seoane-Sepúlveda are supported by CNPq Grant 401735/2013-3 - PVE - Linha 2. 
(1889, 39]), they could have never imagined that, at that precise moment, they had just started a "revolution" in Functional Analysis (we refer to 42] for a detailed and historical exposition). This tool is a fundamental inequality between integrals and an indispensable tool for the study of, among others, $L_{p}$ spaces. Let us recall the classical $L_{p}$ version of this inequality.

Theorem 1.1 (Hölder's inequality, 1889). Let $(\Omega, \Sigma, \mu)$ be a measure space and let $p, q \in[1, \infty]$ with $1 / p+1 / q=1$ (Hölder's conjugates). Then, for all measurable real or complex valued functions $f$ and $g$ on $\Omega$,

$$
\int|f g| d \mu \leq\left(\int|f|^{p} d \mu\right)^{1 / p}\left(\int|g|^{q} d \mu\right)^{1 / q}
$$

If one has $p, q \in(1, \infty), f \in L_{p}(\mu)$, and $g \in L_{q}(\mu)$, then this inequality becomes an equality if and only if $|f|^{p}$ and $|g|^{q}$ are linearly dependent in $L_{1}(\mu)$. When one has $p=q=2$ we recover a form of the Cauchy-Schwarz inequality (or the Cauchy-Bunyakovsky-Schwarz inequality). Also, Hölder's inequality is used to prove Minkowski's inequality (the triangle inequality for $L_{p}$ spaces) and to establish that $L_{q}(\mu)$ is the dual space of $L_{p}(\mu)$ for $p \in[1, \infty)$. Of course, we are all familiar with these classical applications of Hölder's inequality.

As it happens to almost every important result in mathematics, several extensions and generalizations of it have appeared along the time; and in the case of Hölder's inequality, this is not different. One of the extensions is the variant of Hölder's inequality for mixed $L_{p}$ spaces. This inequality appeared in 1961, in the work of A. Benedek and R. Panzone 8. Mixed $L_{p}$ spaces may be seen as a pure exercise of abstraction of the original notion of $L_{p}$ spaces, but as a matter of fact we shall show that the theory developed in [8] plays a crucial role in applications to quite different frameworks; it is intriguing that, although widely known (the paper [8] has more than 100 citations, mainly related to PDEs; we refer, for instance to [20, 34] ) it was overlooked in important fields of mathematics. This gap began to be filled in 2012-2013, when Hölder's inequality for mixed $L_{p}$ spaces was re-discovered as an interpolation-type result and we shall show that different fields of Mathematics and even of Physics were positively influenced. This expository paper is arranged as follows. Section 2 presents some motivation to illustrate the subject of this article. Section 3 is devoted to the aforementioned variant of Hölder's inequality (Hölder's inequality for mixed sums) with a short proof. This result was only written in a proper and organized fashion in 1961 (8]) but, as it will be clear along this paper, at least in the topics gathered here (Functional Analysis, Complex Analysis and Quantum Information Theory) it was surely not been taken advantage of before 2012. Our approach is quite different from the one employed in [8] and we shall follow the lines of [7]. Section 4 will recall several useful inequalities that we shall need and Section 5 is devoted to the recent applications of Hölder's inequality for mixed sums in Functional Analysis and Quantum Information Theory, culminating with the solution of a classical problem from Complex Analysis:

the Bohr radius problem. Applications to the improvement of the constants of the Hardy-Littlewood inequality and separately summing operators are also given.

\section{Motivation: SOME Interpolative PUZZLES}

As a motivation to the subject treated here, let us suppose that we have the following two inequalities at hand, for certain complex scalar matrix $\left(a_{i j}\right)_{i, j=1}^{N}$ : 


$$
\sum_{i=1}^{N}\left(\sum_{j=1}^{N}\left|a_{i j}\right|^{2}\right)^{\frac{1}{2}} \leq \mathrm{C} \text { and } \sum_{j=1}^{N}\left(\sum_{i=1}^{N}\left|a_{i j}\right|^{2}\right)^{\frac{1}{2}} \leq \mathrm{C}
$$

for some constant $\mathrm{C}>0$ and all positive integers $N$.

How can one find an optimal exponent $r$ and a constant $\mathrm{C}_{1}>0$ such that

$$
\left(\sum_{i, j=1}^{N}\left|a_{i j}\right|^{r}\right)^{\frac{1}{r}} \leq \mathrm{C}_{1}
$$

for all positive integers $N$ ? Moreover, how can one get a good (small) constant $\mathrm{C}_{1}$ ?

This question (at least concerning the exponent $r$ can be solved in no less than two ways: interpolation and Hölder's inequality).

First note that, by using a consequence of Minkowski's inequality (see [35]), we know that

$$
\left(\sum_{i=1}^{N}\left(\sum_{j=1}^{N}\left|a_{i j}\right|\right)^{2}\right)^{\frac{1}{2}} \leq \sum_{j=1}^{N}\left(\sum_{i=1}^{N}\left|a_{i j}\right|^{2}\right)^{\frac{1}{2}} \leq \mathrm{C} .
$$

If we use Hölder's inequality twice, we proceed as follows:

$$
\begin{aligned}
\sum_{i, j=1}^{N}\left|a_{i j}\right|^{\frac{4}{3}} & =\sum_{i=1}^{N}\left(\sum_{j=1}^{N}\left|a_{i j}\right|^{\frac{2}{3}}\left|a_{i j}\right|^{\frac{2}{3}}\right) \\
& \leq \sum_{i=1}^{N}\left(\left(\sum_{j=1}^{N}\left|a_{i j}\right|^{2}\right)^{\frac{1}{3}}\left(\sum_{j=1}^{N}\left|a_{i j}\right|\right)^{\frac{2}{3}}\right) \\
& \leq\left(\sum_{i=1}^{N}\left(\sum_{j=1}^{N}\left|a_{i j}\right|^{2}\right)^{\frac{1}{2}}\right)^{\frac{2}{3}}\left(\sum_{i=1}^{N}\left(\sum_{j=1}^{N}\left|a_{i j}\right|\right)^{2}\right)^{\frac{1}{3}} \\
& =\left[\sum_{i=1}^{N}\left(\sum_{j=1}^{N}\left|a_{i j}\right|^{2}\right)^{\frac{1}{2}}\right]^{\frac{2}{3}}\left[\left(\sum_{i=1}^{N}\left(\sum_{j=1}^{N}\left|a_{i j}\right|\right)^{2}\right)^{\frac{1}{2}}\right]^{\frac{2}{3}} \leq \mathrm{C}^{\frac{4}{3}} .
\end{aligned}
$$

By complex interpolation (see [9]) the solution is shorter; essentially we have two mixed inequalities with exponents $(1,2)$ in equation (2.1) and $(2,1)$ in equation (2.2). By interpolating these exponents with $\theta_{1}=\theta_{2}=1 / 2$ we obtain an exponent $(4 / 3,4 / 3)$ with constant C.

The optimality of the exponent $4 / 3$ can be proved using the Kahane-SalemZygmund inequality (Theorem 5.1).

The use of Hölder's inequality as above becomes almost impossible if we have, for instance, 10 sums, 100 sums, etc. The reader can test the case of three sums using Hölder's inequality. More precisely, as a simple illustration suppose that 


$$
\sum_{\sigma(i)=1}^{N}\left(\sum_{\sigma(j)=1}^{N} \sum_{\sigma(k)=1}^{N}\left|a_{i j k}\right|^{2}\right)^{\frac{1}{2}} \leq \mathrm{C}
$$

for all bijections $\sigma:\{i, j, k\} \rightarrow\{i, j, k\}$ and all $N$. How can we find an optimal exponent $r$ and a constant $\mathrm{C}_{1}$ such that

$$
\left(\sum_{i, j, k=1}^{N}\left|a_{i j k}\right|^{r}\right)^{\frac{1}{r}} \leq \mathrm{C}_{1}
$$

for every $N$ ?

The search for good constants dominating the respective inequalities is highly important for applications (see Section 5) and has an extra ingredient when we are using the interpolative approach: the main point is that different interpolations may result in the same exponent, but the constants involved differ. Thus, we must investigate what exponents we shall use to interpolate. More precisely, as we will see in Section 5, the Bohnenblust-Hille inequality for 3-linear forms asserts that there is a constant $\mathrm{C}_{3} \geq 1$ such that, for all 3 -linear forms $T: \ell_{\infty}^{N} \times \ell_{\infty}^{N} \times \ell_{\infty}^{N} \rightarrow \mathbb{K}$,

$$
\left(\sum_{i_{1}, i_{2}, i_{3}=1}^{N}\left|T\left(e_{i_{1}}, e_{i_{2}}, e_{i_{3}}\right)\right|^{\frac{3}{2}}\right)^{\frac{2}{3}} \leq \mathrm{C}_{3}\|T\|
$$

and all $N$, where

$$
\|T\|:=\sup _{\left\|z^{(1)}\right\|=1, \ldots,\left\|z^{(m)}\right\|=1}\left|T\left(z^{(1)}, \ldots, z^{(m)}\right)\right| \quad \text { for all } m \in \mathbb{N} .
$$

However, the exponent $3 / 2$ can be obtained by a "multiple" interpolation of exponents of inequalities of the form

$$
\left(\sum_{i_{1}=1}^{N}\left(\sum_{i_{2}=1}^{N}\left(\sum_{i_{3}=1}^{N}\left|T\left(e_{i_{1}}, e_{i_{2}}, e_{i_{3}}\right)\right|^{q_{3}}\right)^{\frac{q_{2}}{q_{3}}}\right)^{\frac{q_{1}}{q_{2}}} \leq \mathrm{C}\|T\|,\right.
$$

with

$$
\left(q_{1}, q_{2}, q_{3}\right)=(1,2,2),(2,1,2) \text { and }(2,2,1)
$$

or

$$
\left(q_{1}, q_{2}, q_{3}\right)=\left(\frac{4}{3}, \frac{4}{3}, 2\right),\left(\frac{4}{3}, 2, \frac{4}{3}\right) \text { and }\left(2, \frac{4}{3}, \frac{4}{3}\right)
$$

and the last procedure provides quite better constants. This is a simple illustration of the core of the new advances that lead to the results reported in this expository paper.

The theory of $L_{p}$ spaces with mixed norms seems to have been created in 1961, [8], including a Hölder inequality in this framework. However, as we describe along this paper the full strength of this inequality was overlooked and very recently important advances in different fields of Mathematics and Physics were achieved with the help of this Hölder inequality (also called interpolative approach). 


\section{HöLDER's INEQUALITY REVISITED}

Essentially, the simplest version of the Hölder inequality asserts that if $1 / p+$ $1 / q=1$ and $\left(a_{j}\right) \in \ell_{p},\left(b_{j}\right) \in \ell_{q}$ then $\left(a_{j} b_{j}\right) \in \ell_{1}$. In this section we present a variation of this result, which was apparently overlooked in Functional Analysis (but not in PDEs) in the last decades. This variant is a key result of a number of important recent advances in Mathematical Analysis and Mathematical Physics that appeared in the last few years.

The previous result may have been seen as a variant of the following general Hölder's inequality presented in the classical paper [8] on mixed norms in $L_{p}$ spaces. We shall now work with $L_{p}(\mathbb{N})=\ell_{p}$, since it is the case we are interested in. We need to recall some useful multi-index notation: for a positive integer $m$ and a non-void subset $D \subset \mathbb{N}$ we denote the set of multi-indices $\mathbf{i}=\left(i_{1}, \ldots, i_{m}\right)$, with each $i_{k} \in D$, by

$$
\mathcal{M}(m, D):=\left\{\mathbf{i}=\left(i_{1}, \ldots, i_{m}\right) \in \mathbb{N}^{m} ; i_{k} \in D, k=1, \ldots, m\right\}=D^{m} .
$$

We also denote

$$
\mathcal{M}(m, n):=\mathcal{M}(m,\{1,2, \ldots, n\}) .
$$

For $\mathbf{p}=\left(p_{1}, \ldots, p_{m}\right) \in[1, \infty)^{m}$, and a Banach space $X$, let us consider the space

$$
\ell_{\mathbf{p}}(X):=\ell_{p_{1}}\left(\ell_{p_{2}}\left(\ldots\left(\ell_{p_{m}}(X)\right) \ldots\right)\right)
$$

namely, a vector matrix $\left(x_{\mathbf{i}}\right)_{\mathbf{i} \in \mathcal{M}(m, \mathbb{N})} \in \ell_{\mathbf{p}}(X)$ if, and only if,

$$
\left(\sum_{i_{1}=1}^{\infty}\left(\sum_{i_{2}=1}^{\infty}\left(\ldots\left(\sum_{i_{m-1}=1}^{\infty}\left(\sum_{i_{m}=1}^{\infty}\left\|x_{\mathbf{i}}\right\|_{X}^{p_{m}}\right)^{\frac{p_{m}-1}{p_{m}}}\right)^{\frac{p_{m-2}}{p_{m-1}}} \ldots\right)^{\frac{p_{2}}{p_{3}}}\right)^{\frac{p_{1}}{p_{2}}}\right)^{\frac{1}{p_{1}}}<\infty .
$$

When $X=\mathbb{K}$, we just write $\ell_{\mathbf{p}}$ instead of $\ell_{\mathbf{p}}(\mathbb{K})$.

Also, we deal with the coordinatewise product of two scalar matrices $\mathbf{a}=$ $\left(a_{\mathbf{i}}\right)_{\mathbf{i} \in \mathcal{M}(m, n)}$ and $\mathbf{b}=\left(b_{\mathbf{i}}\right)_{\mathbf{i} \in \mathcal{M}(m, n)}$, i.e.,

$$
\mathbf{a b}:=\left(a_{\mathbf{i}} b_{\mathbf{i}}\right)_{\mathbf{i} \in \mathcal{M}(m, n)} .
$$

The following result seems to be first observed by A. Benedek and R. Panzone (see [] $)$ :

Theorem 3.1 (Hölder's inequality for mixed $\ell_{\mathbf{p}}$ spaces). Let $\mathbf{r}, \mathbf{q}(1), \ldots, \mathbf{q}(N) \in$ $[1, \infty]^{m}$ such that

$$
\frac{1}{r_{j}}=\frac{1}{q_{j}(1)}+\cdots+\frac{1}{q_{j}(N)}, \quad j \in\{1,2, \ldots, m\}
$$

and let $\mathbf{a}_{k}, k=1, \ldots, N$ be scalar $m$-square matrix. Then

$$
\left\|\prod_{k=1}^{N} \mathbf{a}_{k}\right\|_{\mathbf{r}} \leq \prod_{k=1}^{N}\left\|\mathbf{a}_{k}\right\|_{\mathbf{q}(k)} .
$$

Remember that, the previous inequality means the following: 


$$
\begin{aligned}
& \left(\sum_{i_{1}=1}^{n}\left(\ldots\left(\sum_{i_{m}=1}^{n}\left|a_{\mathbf{i}}^{1} \cdot a_{\mathbf{i}}^{2} \cdot \ldots \cdot a_{\mathbf{i}}^{N}\right|^{q_{m}}\right)^{\frac{q_{m}-1}{q_{m}}} \ldots\right)^{\frac{q_{1}}{q_{2}}}\right)^{\frac{1}{q_{1}}} \\
& \leq \prod_{k=1}^{N}\left[\left(\sum_{i_{1}=1}^{n}\left(\ldots\left(\sum_{i_{m}=1}^{n}\left|a_{\mathbf{i}}\right|^{q_{m}(k)}\right)^{\frac{q_{m-1}(k)}{q_{m}(k)}} \ldots\right)^{\frac{q_{1}(k)}{q_{2}(k)}}\right)^{\frac{1}{q_{1}(k)}}\right],
\end{aligned}
$$

Using the above result we are able to recover the interpolative inequality from 24,7] (Theorem 3.2 below), that we can also, in some sense, call Hölder's inequality for multiple exponents. Under the point of view of interpolation theory it is not a complicated result but, just in 2013, it began to be used in all its full strength. Its applications (in different fields) are impressive, as we shall illustrate in the remaining of the paper. Just before that, for a positive real number $\theta$, let us define $\mathbf{a}^{\theta}:=\left(a_{\mathbf{i}}^{\theta}\right)_{\mathbf{i} \in \mathcal{M}(m, n)}$. It is straightforward to see that

$$
\left\|\mathbf{a}^{\theta}\right\|_{\mathbf{q} / \theta}=\|\mathbf{a}\|_{\mathbf{q}}^{\theta}
$$

where $\mathbf{q} / \theta:=\left(q_{1} / \theta, \ldots, q_{m} / \theta\right)$.

Theorem 3.2 (Hölder's inequality for multiple exponents -interpolative approach-).

Let $m, n, N$ be positive integers and $\mathbf{q}, \mathbf{q}(1), \ldots, \mathbf{q}(N) \in[1, \infty)^{m}$ be such that $\left(\frac{1}{q_{1}}, \ldots, \frac{1}{q_{m}}\right)$

belongs to the convex hull of $\left(\frac{1}{q_{1}(k)}, \ldots, \frac{1}{q_{m}(k)}\right), k=1, \ldots, N$. Then for all scalar $\operatorname{matrix} \mathbf{a}=\left(a_{\mathbf{i}}\right)_{\mathbf{i} \in \mathcal{M}(m, n)}$,

i.e.,

$$
\|\mathbf{a}\|_{\mathbf{q}} \leq \prod_{k=1}^{N}\|\mathbf{a}\|_{\mathbf{q}(k)}^{\theta_{k}}
$$

$$
\begin{aligned}
& \left(\sum_{i_{1}=1}^{n}\left(\ldots\left(\sum_{i_{m}=1}^{n}\left|a_{\mathbf{i}}\right|^{q_{m}}\right)^{\frac{q_{m-1}}{q_{m}}} \ldots\right)^{\frac{q_{1}}{q_{2}}}\right)^{\frac{1}{q_{1}}} \\
\leq & \prod_{k=1}^{N}\left[\left(\sum_{i_{1}=1}^{n}\left(\ldots\left(\sum_{i_{m}=1}^{n}\left|a_{\mathbf{i}}\right|^{q_{m}(k)}\right)^{\frac{q_{m-1}(k)}{q_{m}(k)}} \ldots\right)^{\frac{q_{1}(k)}{q_{2}(k)}}\right)^{\frac{1}{q_{1}(k)}}\right]^{\theta_{k}},
\end{aligned}
$$

where $\theta_{k}$ are the coordinates of $\left(\frac{1}{q_{1}(k)}, \ldots, \frac{1}{q_{m}(k)}\right)$ on the convex hull.

Proof. For $j=1, \ldots, m$ we have

$$
\frac{1}{q_{j}}=\frac{\theta_{1}}{q_{j}(1)}+\ldots+\frac{\theta_{N}}{q_{j}(N)}=\frac{1}{q_{j}(1) / \theta_{1}}+\ldots+\frac{1}{q_{j}(N) / \theta_{N}} .
$$

Since $\left\|\mathbf{a}^{\theta_{k}}\right\|_{\mathbf{q}(k) / \theta_{k}}=\|\mathbf{a}\|_{\mathbf{q}(k)}^{\theta_{k}}$, by the Hölder inequality for mixed $\ell_{\mathbf{p}}$ spaces we conlude that

$$
\|\mathbf{a}\|_{\mathbf{q}}=\left\|\mathbf{a}^{\theta_{1}+\cdots+\theta_{N}}\right\|_{\mathbf{q}}=\left\|\prod_{k=1}^{N} \mathbf{a}^{\theta_{k}}\right\|_{\mathbf{q}} \leq \prod_{k=1}^{N}\left\|\mathbf{a}^{\theta_{k}}\right\|_{\mathbf{q}(k) / \theta_{k}}=\prod_{k=1}^{N}\|\mathbf{a}\|_{\mathbf{q}(k)}^{\theta_{k}} .
$$


For the sake of completeness of this article, we would also like to present the following proof, which is based on interpolation.

(Interpolative Approach). We just follow the lines of [2, Proposition 2.1]. Proceeding by induction on $N$ and using that, for any Banach space $X$ and $\theta \in[0,1]$,

$$
\ell_{\mathbf{r}}(X)=\left[\ell_{\mathbf{p}}(X), \ell_{\mathbf{q}}(X)\right]_{\theta},
$$

with $\frac{1}{r_{i}}=\frac{\theta}{p_{i}}+\frac{1-\theta}{q_{i}}$, for $i=1, \ldots, m$ (see [9]). If

$$
\frac{1}{q_{i}}=\frac{\theta_{1}}{q_{i}(1)}+\cdots+\frac{\theta_{N}}{q_{i}(N)}
$$

with $\sum_{k=1}^{N} \theta_{k}=1$ and each $\theta_{k} \in[0,1]$, then we also have

$$
\frac{1}{q_{i}}=\frac{\theta_{1}}{q_{i}(1)}+\frac{1-\theta_{1}}{p_{i}}
$$

setting

$$
\frac{1}{p_{i}}=\frac{\alpha_{2}}{q_{i}(2)}+\cdots+\frac{\alpha_{N}}{q_{i}(N)}, \quad \text { and } \alpha_{j}=\frac{\theta_{j}}{1-\theta_{1}},
$$

for $i=1, \ldots, m$ and $j=2, \ldots, N$. So $\alpha_{j} \in[0,1]$ and $\sum_{j=2}^{N} \alpha_{j}=1$. Therefore, by the induction hypothesis, we conclude that

$$
\|\mathbf{a}\|_{\mathbf{q}} \leq\|\mathbf{a}\|_{\mathbf{q}(1)}^{\theta_{1}} \cdot\|\mathbf{a}\|_{\mathbf{p}}^{1-\theta_{1}} \leq\|\mathbf{a}\|_{\mathbf{q}(1)}^{\theta_{1}} \cdot\left[\prod_{j=2}^{N}\|\mathbf{a}\|_{\mathbf{q}(j)}^{\alpha_{j}}\right]^{1-\theta_{1}}=\prod_{k=1}^{N}\|\mathbf{a}\|_{\mathbf{q}(k)}^{\theta_{k}} .
$$

Combining the previous result with Minkowski's inequality we have a very useful inequality (see [7, Remark 2.2]):

Corollary 3.3. Let $m, n$ be positive integers, $1 \leq k \leq m$ and $1 \leq s \leq q$. Then for all scalar matrix $\left(a_{\mathbf{i}}\right)_{\mathbf{i} \in \mathcal{M}(m, n)}$,

$$
\left(\sum_{\mathbf{i} \in \mathcal{M}(m, n)}\left|a_{\mathbf{i}}\right|^{\rho}\right)^{\frac{1}{\rho}} \leq \prod_{S \in \mathcal{P}_{k}(m)}\left(\sum_{\mathbf{i}_{S}}\left(\sum_{\mathbf{i}_{\widehat{S}}}\left|a_{\mathbf{i}}\right|^{q}\right)^{\frac{s}{q}}\right)^{\frac{1}{s} \cdot \frac{1}{\left(\begin{array}{c}
m \\
k
\end{array}\right)}},
$$

where

$$
\rho:=\frac{m s q}{k q+(m-k) s}
$$

and $\mathcal{P}_{k}(m)$ stands for the set of subsets $S \subseteq\{1, \ldots, m\}$ with $\operatorname{card}(S)=k$.

The above corollary shows that Blei's inequality (see Corollary 3.4 below) is just a very particular case of a huge family of similar inequalities. For our purposes, the crucial point is that the use of Blei's inequality is far from being a good option to obtain good estimates for the constants of the Bohnenblust-Hille and related inequalities. Just to illustrate the strength of Theorem 3.2 and Corollary 3.3 we present here a very simple proof (see [7]) of Blei's inequality. 
Corollary 3.4 (Blei's inequality - Defant, Popa, Schwarting approach, 25]). Let $A$ and $B$ be two finite non-void index sets. Let $\left(a_{i j}\right)_{(i, j) \in A \times B}$ be a scalar matrix with positive entries, and denote its columns by $\alpha_{j}=\left(a_{i j}\right)_{i \in A}$ and its rows by $\beta_{i}=\left(a_{i j}\right)_{j \in B}$. Then, for $q, s_{1}, s_{2} \geq 1$ with $q>\max \left(s_{1}, s_{2}\right)$ we have

$$
\left(\sum_{(i, j) \in A \times B} a_{i j}^{w\left(s_{1}, s_{2}\right)}\right)^{\frac{1}{w\left(s_{1}, s_{2}\right)}} \leq\left(\sum_{i \in A}\left\|\beta_{i}\right\|_{q}^{s_{1}}\right)^{\frac{f\left(s_{1}, s_{2}\right)}{s_{1}}}\left(\sum_{j \in B}\left\|\alpha_{j}\right\|_{q}^{s_{2}}\right)^{\frac{f\left(s_{2}, s_{1}\right)}{s_{2}}},
$$

with

$$
\begin{gathered}
w:[1, q)^{2} \rightarrow[0, \infty), w(x, y):=\frac{q^{2}(x+y)-2 q x y}{q^{2}-x y}, \\
f:[1, q)^{2} \rightarrow[0, \infty), f(x, y):=\frac{q^{2} x-q x y}{q^{2}(x+y)-2 q x y} .
\end{gathered}
$$

Proof. Let us consider the exponents

$$
\left(q, s_{2}\right),\left(s_{1}, q\right)
$$

and

$$
\left(\theta_{1}, \theta_{2}\right)=\left(f\left(s_{2}, s_{1}\right), f\left(s_{1}, s_{2}\right)\right) .
$$

Note that $\left(w\left(s_{1}, s_{2}\right), w\left(s_{1}, s_{2}\right)\right)$ is obtained by interpolating $\left(q, s_{2}\right)$ and $\left(s_{1}, q\right)$ with $\theta_{1}, \theta_{2}$, respectively. Then, from Theorem 3.2 , we have

$$
\left(\sum_{(i, j) \in A \times B} a_{i j}^{w\left(s_{1}, s_{2}\right)}\right)^{\frac{1}{w\left(s_{1}, s_{2}\right)}} \leq\left(\sum_{i \in A}\left\|\beta_{i}\right\|_{q}^{s_{1}}\right)^{\frac{f\left(s_{1}, s_{2}\right)}{s_{1}}}\left(\sum_{i \in A}\left\|\beta_{i}\right\|_{s_{2}}^{q}\right)^{\frac{f\left(s_{2}, s_{1}\right)}{q}} .
$$

Now, since $q>s_{2}$ we just need to use Propositon 4.6 to change the order of the last sum.

We invite the interest reader to compare the above proof with the proof presented in [25, pages 226-227], in which the classical Hölder's inequality is needed several times.

\section{Some useful inequalities}

The main recent advances presented here are direct or indirect consequence of the improvements obtained in the polynomial and multilinear Bohnenblust-Hille inequalities (and these improvements were obtained by using the theory of mixed $L_{p}$ spaces and the core of the results lie in the variant of Hölder's inequality (Theorem 3.2). However we also need three other important ingredients: the Khinchine inequality (and its version for multiple sums), Kahane-Salem-Zygmund's inequality in its polynomial and multilinear versions and a variant of Minkowski's inequality. Before that, let us provide a brief account on polynomials and multilinear operators, that shall be needed in the remaining of the article.

Polynomials in Banach spaces (at least for complex scalars) are of fundamental importance in the theory of Infinite Dimensional Holomorphy (see [30, 45]). In general the theory of polynomials and multilinear operators between normed spaces has its importance in different areas of Mathematics, from Number Theory, or Dirichlet series, to Functional Analysis.

In this section we recall the concepts of polynomials and multilinear operators between Banach spaces and some "folkloric results", that will be needed here. 
If $E_{1}, \ldots, E_{m}$, and $F$ are vector spaces, a $m$-linear operator $T: E_{1} \times \cdots \times E_{m} \rightarrow F$ is a map that is linear in each coordinate separately. When $E_{1}=\cdots=E_{m}=E$ we say that $T$ is symmetric if $T\left(x_{\sigma(1)}, \ldots, x_{\sigma(m)}\right)=T\left(x_{1}, \ldots, x_{m}\right)$ for all bijections $\sigma:\{1, \ldots, m\} \rightarrow\{1, \ldots, m\}$.

If $E, F$ are vector spaces, a $m$-homogeneous polynomial is a map $P: E \rightarrow F$ such that

$$
P(x)=T(x, \ldots, x)
$$

for some $m$-linear operator $T: E \times \cdots \times E \rightarrow F$. Continuity is defined is the obvious fashion.

Fixed $E, F, E_{1}, \ldots, E_{m}$, the spaces of continuous $m$-homogeneous polynomials from $E$ to $F$ are represented by $\mathcal{P}\left({ }^{m} E ; F\right)$ and the space of continuous multilinear operators from $E_{1} \times \cdots \times E_{m}$ to $F$ is denoted by $\mathcal{L}\left(E_{1}, \ldots, E_{m} ; F\right)$. Both vector spaces are Banach spaces when endowed with the sup norm in the unit ball of $B_{E}$ or in product of the the unit balls $B_{E_{1}} \times \cdots \times B_{E_{m}}$.

The following characterizations of continuous polynomials are elementary (analogous results holds for multilinear operators):

Proposition 4.1. Let $E, F$ be vector spaces, $P \in \mathcal{P}\left({ }^{m} E ; F\right)$. The following assertions are equivalent:

(i) $P \in \mathcal{P}\left({ }^{m} E ; F\right)$;

(ii) $P$ is continuous at zero;

(iii) There is a constant $\mathrm{M}>0$ such that $\|P(x)\| \leq \mathrm{M}\|x\|^{m}$, for all $x \in E$;

The Polarization Formula relates polynomials and symmetric multilinear operators in a very useful way. Its proof is a kind of consequence of the Leibnitz formula and some combinatorial tricks (see [30,45]).

Theorem 4.2 (Polarization Formula). Let $E, F$ be linear spaces. If $T \in \mathcal{L}\left({ }^{m} E ; F\right)$ is symmetric then

$$
T\left(x_{1}, \ldots, x_{m}\right)=\frac{1}{m ! 2^{m}} \sum_{\varepsilon_{i}= \pm 1} \varepsilon_{1} \cdots \varepsilon_{m} T\left(x_{0}+\varepsilon_{1} x_{1}+\cdots+\varepsilon_{m} x_{m}\right)^{m},
$$

for all $x_{0}, x_{1}, x_{2}, \ldots, x_{m} \in E$.

The following result is an immediate consequence of the Polarization Formula:

Corollary 4.3. For each $m$-homogeneous polynomial there is a unique m-linear operator associated to it. In other words, if $P$ is a m-homogeneous polynomial, then there exists only one symmetric $m$-linear operator $T$ (sometimes called polar of $P$ ) such that

$$
P(x)=T(x, \ldots, x)
$$

for all $x$.

In general, if $T$ is the symmetric $m$-linear operator associated to a $m$-homogeneous polynomial $P$ we have

$$
\|P\| \leq \frac{m^{m}}{m !}\|T\|
$$

where $\|P\|=\sup _{\|z\|=1}|P(z)|$. The constant $\frac{m^{m}}{m !}$ is usually called polarization constant. 
If $P$ is a homogeneous polynomial of degree $m$ on $\mathbb{K}^{n}$ given by

$$
P\left(x_{1}, \ldots, x_{n}\right)=\sum_{|\alpha|=m} a_{\alpha} \mathbf{x}^{\alpha},
$$

and $L$ is the polar of $P$, then

$$
L\left(e_{1}^{\alpha_{1}}, \ldots, e_{n}^{\alpha_{n}}\right)=\frac{a_{\alpha}}{\left(\begin{array}{c}
m \\
\alpha
\end{array}\right)},
$$

where $\left\{e_{1}, \ldots, e_{n}\right\}$ is the canonical basis of $\mathbb{K}^{n}$ and $e_{k}^{\alpha_{k}}$ stands for $e_{k}$ repeated $\alpha_{k}$ times, the $\alpha_{j}$ 's are non negative integers with $\sum_{j=1}^{n} \alpha_{j}=\alpha$, and $\mathbf{x}^{\alpha}=x_{1}^{\alpha_{1}} \cdot \ldots \cdot x_{n}^{\alpha_{n}}$.

4.1. Khinchine's inequality. The Khinchine inequality in its modern presentation has its roots in [51]. Let $\left(\varepsilon_{i}\right)_{i \geq 1}$ be a sequence of independent Rademacher variables. Then, for any $p \in[1,2]$, there exists a constant $\mathrm{A}_{\mathbb{R}, p}$ such that, for any sequence $\left(a_{i}\right)$ of real numbers with finite support,

$$
\left(\sum_{i=1}^{\infty}\left|a_{i}\right|^{2}\right)^{1 / 2} \leq \mathrm{A}_{\mathbb{R}, p}^{-1}\left(\int_{[0,1]^{m}}\left|\sum_{i=1}^{\infty} a_{i} \varepsilon_{i}(\omega)\right|^{p} d \omega\right)^{1 / p} .
$$

For complex scalars it more useful (since it gives better constants) to use the following version of Khinchine's inequality (called Khinchine's inequality with Steinhaus variables): for any $p \in[1,2]$, there exists a constant $\mathrm{A}_{\mathbb{C}, p}$ such that, for any sequence $\left(a_{i}\right)$ of complex numbers with finite support

$$
\left(\sum_{i=1}^{\infty}\left|a_{i}\right|^{2}\right)^{1 / 2} \leq \mathrm{A}_{\mathbb{C}, p}^{-1}\left(\int_{\mathbb{T} \infty}\left|\sum_{i=1}^{\infty} a_{i} z_{i}\right|^{p} d z\right)^{1 / p}
$$

with $\mathbb{T}^{\infty}$ denoting the infinite polycircle, i.e.,

$$
\mathbb{T}^{\infty}=\left\{z=\left(z_{i}\right)_{i \in \mathbb{N}} \in \mathbb{C}^{\mathbb{N}}:\left|z_{i}\right|=1 \text { for all } i \in \mathbb{N}\right\},
$$

and $d z$ denoting the standard Lebesgue probability measure on $\mathbb{T}^{\infty}$. The best constants $\mathrm{A}_{\mathbb{R}, p}$ and $\mathrm{A}_{\mathbb{C}, p}$ were obtained by Haagerup and König, respectively (see [36] and [41]). More precisely,

- $\mathrm{A}_{\mathbb{R}, p}=\frac{1}{\sqrt{2}}\left(\frac{\Gamma\left(\frac{1+p}{2}\right)}{\sqrt{\pi}}\right)^{1 / p}$ if $p>p_{0} \approx 1.8474$;

- $\mathrm{A}_{\mathbb{R}, p}=2^{\frac{1}{2}-\frac{1}{p}}$ if $p<p_{0}$;

- $\mathrm{A}_{\mathbb{C}, p}=\Gamma\left(\frac{p+2}{2}\right)^{1 / p}$ if $p \in[1,2]$.

The (apparently) strange value $p_{0} \approx 1.8474$ is, to be precise, the unique number $p_{0} \in(1,2)$ with

$$
\Gamma\left(\frac{p_{0}+1}{2}\right)=\frac{\sqrt{\pi}}{2} .
$$

The notation $A_{\mathbb{K}, p}$ will be kept along this paper.

Using Fubini's theorem and Minkowski's inequality (see, for instance, 25, Lemma $2.2]$ for the real case and [46, Theorem 2.2] for the complex case), these inequalities have a multilinear version: for any $n, m \geq 1$, for any family $\left(a_{\mathbf{i}}\right)_{\mathbf{i} \in \mathbb{N} m}$ of real (resp. complex) numbers with finite support,

$$
\left(\sum_{\mathbf{i} \in \mathbb{N}^{m}}\left|a_{\mathbf{i}}\right|^{2}\right)^{1 / 2} \leq \mathrm{A}_{\mathbb{R}, p}^{-m}\left(\int_{[0,1]^{m}}\left|\sum_{\mathbf{i} \in \mathbb{N}^{m}} a_{\mathbf{i}} \varepsilon_{i_{1}}^{(1)}\left(\omega_{1}\right) \ldots \varepsilon_{i_{m}}^{(m)}\left(\omega_{m}\right)\right|^{p} d \omega_{1} \cdots d \omega_{m}\right)^{1 / p}
$$


where $\left(\varepsilon_{i}^{(1)}\right), \ldots,\left(\varepsilon_{i}^{(m)}\right)$ are sequences of independent Rademacher variables (resp.

$$
\left(\sum_{\mathbf{i} \in \mathbb{N}^{m}}\left|a_{\mathbf{i}}\right|^{2}\right)^{1 / 2} \leq \mathrm{A}_{\mathbb{C}, p}^{-m}\left(\int_{(\mathbb{T} \infty)^{m}}\left|\sum_{\mathbf{i} \in \mathbb{N}^{m}} a_{\mathbf{i}} z_{i_{1}}^{(1)} \ldots z_{i_{m}}^{(m)}\right|^{p} d z^{(1)} \ldots d z^{(m)}\right)^{1 / p},
$$

in the complex case).

4.2. Kahane-Salem-Zygmund's inequality. The essence of the Kahane-SalemZygmund inequalities, as we describe below, probably appeared for the first time in 40], but our approach follows the lines of Boas' paper [11. Paraphrasing Boas, the Kahane-Salem-Zygmund inequalities use probabilistic methods to construct a homogeneous polynomial (or multilinear operator) with a relatively small supremum norm but relatively large majorant function. Both the multilinear and polynomial versions are needed for our goals.

Theorem 4.4 (Kahane-Salem-Zygmund's inequality - Multilinear version, 11]). Let $m, n$ be positive integers. There exists a m-linear map $T_{m, n}: \ell_{\infty}^{n} \times \cdots \times \ell_{\infty}^{n} \rightarrow \mathbb{K}$ of the form

$$
T_{m, n}\left(z^{(1)}, \ldots, z^{(m)}\right)=\sum_{i_{1}, \ldots, i_{m}=1}^{n} \pm z_{i_{1}}^{(1)} \ldots z_{i_{m}}^{(m)}
$$

such that

$$
\left\|T_{m, n}\right\| \leq \sqrt{32 \log (6 m)} \times n^{\frac{m+1}{2}} \times \sqrt{n !} .
$$

The original version of the Kahane-Salem-Zygmund appears in the framework of complex scalars but it is simple to verify that the same result (with the same constants) holds for real scalars. The folowing result is corollary of the previous, now for polynomials, and it will also be important for our aims.

Theorem 4.5 (Kahane-Salem-Zygmund's inequality - Polynomial version, 11). Let $m, n$ be positive integers. Then there exists a m-homogeneous polynomial $P$ : $\ell_{\infty}^{n} \rightarrow \mathbb{K}$ of the form

$$
P_{m, n}(\mathbf{z})=\sum_{|\alpha|=d} \pm\left(\begin{array}{c}
m \\
\alpha
\end{array}\right) \mathbf{z}^{\alpha}
$$

such that

$$
\left\|P_{m, n}\right\| \leq \sqrt{32 \log (6 m)} \times n^{\frac{m+1}{2}} \times \sqrt{n !} .
$$

4.3. A corollary to Minkowski's inequality. Minkowski's inequality is a very well-known result that helps to prove that $L_{p}$ spaces are Banach spaces: it is the triangle inequality for $L_{p}$ spaces. We need a somewhat well known result, which is a corollary of one of the many versions of Minkowski's inequality, whose proof can be found, for instance, in [35].

Proposition 4.6 (Corollary to Minkowski's inequality). For any $0<p \leq q<\infty$ and for any matrix of complex numbers $\left(c_{i j}\right)_{i, j=1}^{\infty}$,

$$
\left(\sum_{i=1}^{\infty}\left(\sum_{j=1}^{\infty}\left|c_{i j}\right|^{p}\right)^{q / p}\right)^{1 / q} \leq\left(\sum_{j=1}^{\infty}\left(\sum_{i=1}^{\infty}\left|c_{i j}\right|^{q}\right)^{p / q}\right)^{1 / p}
$$




\section{RECENT "UNEXPECTED" APPLICATIONS TO CLASSICAL PROBLEMS}

5.1. The Bohnenblust-Hille inequality with subpolynomial constants. The Riemann hypothesis certainly motivated and inspired many prestigious mathematicians from the 20th century to study Dirichlet sums in a more extensive fashion (for instance, Bourgain, Enflo, or Montgomery [16, 32,44]). Perhaps, for this reason, in the first decades of the 20th century Harald Bohr was merged in the study of Dirichlet series (see 13 15). One of his main interests was to determine the width of the maximal strips on which a Dirichlet series can converge absolutely but non uniformly. More precisely, for a Dirichlet series $\sum_{n} a_{n} n^{-s}$, Bohr defined

$$
\begin{gathered}
\sigma_{a}=\inf \left\{r: \sum_{n} a_{n} n^{-s} \text { converges for } \operatorname{Re}(s)>r\right\}, \\
\sigma_{u}=\inf \left\{r: \sum_{n} a_{n} n^{-s} \text { converges uniformly in } \operatorname{Re}(s)>r+\varepsilon \text { for every } \varepsilon>0\right\},
\end{gathered}
$$

and

$$
T=\sup \left\{\sigma_{a}-\sigma_{u}\right\}
$$

Bohr's question was: What is the value of $T$ ?

The Bohnenblust-Hille inequality was proved in 1931 by H.F. Bohnenblust and E. Hille and it is a crucial tool to answer Bohr's problem: They proved that $T=1 / 2$.

When dealing with the Bohnenblust-Hille inequality it is elucidative to begin by proving Littlewood's $4 / 3$ inequality, a predecessor of the Bohnenblust-Hille inequality. Littlewood's 4/3 inequality was proved in 1930 to solve a problem posed by P.J. Daniell. It is worth noticing how Holder's inequality plays a fundamental role in the argument used in the proof. We include (for the sake of completeness) a proof of the optimality of the power $4 / 3$ using the Kahane-Salem-Zygmund inequality.

Theorem 5.1 (Littlewood's $4 / 3$ inequality). There is a constant $\mathrm{L}_{\mathbb{K}} \geq 1$ such that

$$
\left(\sum_{i, j=1}^{N}\left|U\left(e_{i}, e_{j}\right)\right|^{\frac{4}{3}}\right)^{\frac{3}{4}} \leq \mathrm{L}_{\mathbb{K}}\|U\|
$$

for every bilinear form $U: \ell_{\infty}^{N} \times \ell_{\infty}^{N} \rightarrow \mathbb{K}$ and every positive integer $N$. Moreover, the power $4 / 3$ is optimal.

Proof. Note that

$$
\sum_{i, j=1}^{N}\left|U\left(e_{i}, e_{j}\right)\right|^{\frac{4}{3}} \leq\left[\sum_{i=1}^{N}\left(\sum_{j=1}^{N}\left|U\left(e_{i}, e_{j}\right)\right|^{2}\right)^{\frac{1}{2}}\right]^{\frac{2}{3}}\left[\left(\sum_{i=1}^{N}\left(\sum_{j=1}^{N}\left|U\left(e_{i}, e_{j}\right)\right|\right)^{2}\right)^{\frac{1}{2}}\right]^{\frac{2}{3}}
$$

is a particular case of the procedure from Section 2. Now we just need to estimate the two factors above. From the Khinchine inequality we have 


$$
\begin{aligned}
\sum_{i=1}^{N}\left(\sum_{j=1}^{N}\left|U\left(e_{i}, e_{j}\right)\right|^{2}\right)^{\frac{1}{2}} & \leq \sqrt{2} \sum_{i=1}^{N} \int_{0}^{1}\left|\sum_{j=1}^{N} r_{j}(t) U\left(e_{i}, e_{j}\right)\right| d t \\
& \leq \sqrt{2} \int_{0}^{1} \sum_{i=1}^{N}\left|U\left(e_{i}, \sum_{j=1}^{N} r_{j}(t) e_{j}\right)\right| d t \\
& \leq \sqrt{2} \sup _{t \in[0,1]} \sum_{i=1}^{N}\left|U\left(e_{i}, \sum_{j=1}^{N} r_{j}(t) e_{j}\right)\right| \\
& \leq \sqrt{2}\|U\| .
\end{aligned}
$$

By symmetry, the same is true swapping $i$ and $j$. From Minkowski's inequality we have

$$
\left(\sum_{i=1}^{N}\left(\sum_{j=1}^{N}\left|U\left(e_{i}, e_{j}\right)\right|\right)^{2}\right)^{\frac{1}{2}} \leq \sum_{j=1}^{N}\left(\sum_{i=1}^{N}\left|U\left(e_{i}, e_{j}\right)\right|^{2}\right)^{\frac{1}{2}} \leq \sqrt{2}\|U\|
$$

and combining all these inequalities we obtain the result with

$$
\mathrm{L}_{\mathbb{K}}=\sqrt{2} .
$$

To prove the optimality of the exponent $4 / 3$ we can use the Kahane-Salem-Zygmund inequality. Let $T_{2, N}: \ell_{\infty}^{N} \rightarrow \mathbb{C}$ be the bilinear form satisfying the multilinear Kahane-Salem-Zygmund inequality (Theorem 4.4). Then

$$
\left(\sum_{i, j=1}^{N}\left|T_{2, N}\left(e_{i}, e_{j}\right)\right|^{q}\right)^{\frac{1}{q}} \leq \sqrt{2} \mathrm{C} N^{\frac{3}{2}}
$$

and thus

$$
N^{\frac{2}{q}} \leq \sqrt{2} \mathrm{C} N^{\frac{3}{2}}
$$

Next, letting $N \rightarrow \infty$ we conclude that $q \geq \frac{4}{3}$.

The natural generalization of Littlewood's $4 / 3$ inequality is the BohnenblustHille inequality. This inequality essentially says that for $m>2$ the exponent $\frac{4}{3}$ can be replaced by $\frac{2 m}{m+1}$, and this exponent is optimal. More precisely, it asserts that, for any $m \geq 2$, there exists a constant $\mathrm{C}_{\mathbb{K}, m} \geq 1$ such that, for all $m$-linear forms $T: \ell_{\infty}^{N} \times \cdots \times \ell_{\infty}^{N} \rightarrow \mathbb{K}$

$$
\left(\sum_{i_{1}, \ldots, i_{m}=1}^{N}\left|T\left(e_{i_{1}}, \ldots, e_{i_{m}}\right)\right|^{\frac{2 m}{m+1}}\right)^{\frac{m+1}{2 m}} \leq \mathrm{C}_{\mathbb{K}, m}\|T\|,
$$

and all $N$.

This result was overlooked and, sometimes, rediscovered during the last 80 years. Different approaches led to different values of the constants $\mathrm{C}_{m}$. Let us denote the optimal constants satisfying equation (5.1) above by $\mathrm{B}_{\mathbb{K}, m}^{\mathrm{mult}}$. As a matter of fact, controlling the growth of the constants $\mathrm{B}_{\mathbb{K}, m}^{\mathrm{mult}}$ is crucial for applications, as it is being left clear along the paper. 
Now we show how a suitable use of Hölder's inequality (Theorem 3.2) provides a very simple proof of the Bohnenblust-Hille inequality, with (so far!) the best known constants.

With the ingredients of Section 4 we can easily obtain an inductive formula for $\mathrm{B}_{\mathbb{K}, m}^{\text {mult }}$. We present a sketch of the proof (more details can be found in [7]; we also refer to the excellent survey [28]).

Theorem 5.2 (Bohnenblust-Hille inequality). For any positive integer $m$, there exists a constant $\mathrm{B}_{\mathbb{K}, m}^{\text {mult }} \geq 1$ such that, for all m-linear forms $L: \ell_{\infty}^{N} \times \cdots \times \ell_{\infty}^{N} \rightarrow \mathbb{K}$ and all $N$,

$$
\left(\sum_{i_{1}, \ldots, i_{m}=1}^{N}\left|L\left(e_{i_{1}}, \ldots, e_{i_{m}}\right)\right|^{\frac{2 m}{m+1}}\right)^{\frac{m+1}{2 m}} \leq \mathrm{B}_{\mathbb{K}, m}^{\operatorname{mult}}\|L\|,
$$

with $\mathrm{B}_{\mathbb{K}, 1}^{\mathrm{mult}}=1$ and $\mathrm{B}_{\mathbb{K}, m}^{\mathrm{mult}} \leq \mathrm{A}_{\mathbb{K}, \frac{2 k}{k+1}}^{-1} \mathrm{~B}_{\mathbb{K}, k}^{\text {mult }}$.

Proof. We present a simple proof for the case $k=m-1$, which is the most important, since it provides better constants (and the proof for other values of $k$ is similar). The proof for $\mathbb{R}$ is essentially the same as the proof for $\mathbb{C}$, so we present only the proof for the complex case. Let $n \geq 1$ and let $L=\sum_{\mathbf{i} \in \mathbb{N}^{m}} a_{\mathbf{i}} z_{i_{1}}^{(1)} \ldots z_{i_{m}}^{(m)}$ be an $m$-linear form on $\ell_{\infty}^{N} \times \cdots \times \ell_{\infty}^{N}$.

From the Khinchine inequality we have

$$
\left(\sum_{\mathbf{i}_{S}}\left(\sum_{\mathbf{i}_{\hat{S}}}\left|a_{\mathbf{i}}\right|^{2}\right)^{\frac{1}{2} \times \frac{2 m-2}{m}}\right)^{\frac{m}{2 m-2}} \leq \mathrm{A}_{\mathbb{C}, \frac{2 m-2}{m}}^{-1} \mathrm{~B}_{\mathbb{C}, m-1}^{\text {mult }}\|L\| .
$$

with exponents

$$
\left(q_{1}, \ldots, q_{m}\right)=\left(\frac{2 m-2}{m}, \ldots, \frac{2 m-2}{m}, 2\right)
$$

From the "Minkowski inequality" (Proposition 4.6) we can obtain analogous estimates if we take the 2 in the last position and move it backwards making it take every position from the last to the first; in other words, considering the following exponents:

$$
\left(\frac{2 m-2}{m}, \ldots, 2, \frac{2 m-2}{m}\right), \ldots,\left(2, \frac{2 m-2}{m}, \ldots, \frac{2 m-2}{m}\right)
$$

and the same constant. Using the Hölder inequality for multiple exponents we reach the result.

Using the values of the constants $A_{\mathbb{K}, p}$ we conclude that

$$
\mathrm{B}_{\mathbb{C}, m}^{\mathrm{mult}} \leq \prod_{j=2}^{m} \Gamma\left(2-\frac{1}{j}\right)^{\frac{j}{2-2 j}} .
$$

For real scalars and $m \geq 14$,

$$
\mathrm{B}_{\mathbb{R}, m}^{\mathrm{mult}} \leq 2^{\frac{446381}{55440}-\frac{m}{2}} \prod_{j=14}^{m}\left(\frac{\Gamma\left(\frac{3}{2}-\frac{1}{j}\right)}{\sqrt{\pi}}\right)^{\frac{j}{2-2 j}}
$$


and

$$
\mathrm{B}_{\mathbb{R}, m}^{\mathrm{mult}} \leq \prod_{j=2}^{m} 2^{\frac{1}{2 j-2}}=(\sqrt{2})^{\sum_{j=1}^{m-1} 1 / j}
$$

for $2 \leq m \leq 13$.

However, a first look at (5.3) and (5.4) gives a priori no clues on their behavior. The following consequences of Theorem [5.2 taken from [7] are illuminating:

- There exists $\kappa_{1}>0$ such that, for any $m \geq 1$,

$$
\mathrm{B}_{\mathbb{C}, m}^{\text {mult }} \leq \kappa_{1} m^{\frac{1-\gamma}{2}}<\kappa_{1} m^{0.211392} .
$$

- There exists $\kappa_{2}>0$ such that, for any $m \geq 1$,

$$
\mathrm{B}_{\mathbb{R}, m}^{\text {mult }} \leq \kappa_{2} m^{\frac{2-\log 2-\gamma}{2}}<\kappa_{2} m^{0.36482} .
$$

It is interesting to note that some old estimates $\mathrm{B}_{\mathbb{K}, m}^{\text {mult }}$ can be easily recovered just by choosing different $\left(q_{1}, \ldots, q_{m}\right)$ when using Hölder's inequality (or using Theorem 5.2 directly). For instance,

- Davie $([22,1973)$.

$$
\mathrm{B}_{\mathbb{K}, m}^{\mathrm{mult}} \leq(\sqrt{2})^{m-1}
$$

Using the Khinchine inequality, we have

$$
\left(\sum_{i_{1}=1}^{n}\left(\ldots\left(\sum_{i_{m}=1}^{n}\left|a_{\mathbf{i}}\right|^{q_{m}}\right)^{\frac{q_{m-1}}{q_{m}}} \ldots\right)^{\frac{q_{1}}{q_{2}}} \leq(\sqrt{2})^{\frac{1}{q_{1}}}\|L\|\right.
$$

for

$$
\left(q_{1}, \ldots, q_{m}\right)=(1,2, \ldots, 2)
$$

Using the "Minkowski inequality" (Proposition 4.6) we get the same estimate for

$$
\left(q_{1}, \ldots, q_{m}\right)=(2,1, \ldots, 2), \ldots,\left(q_{1}, \ldots, q_{m}\right)=(2, \ldots, 2,1)
$$

with the same constant. Now, using Theorem 3.2 we conclude the proof with

$$
\mathrm{B}_{\mathbb{K}, m}^{\mathrm{mult}} \leq(\sqrt{2})^{m-1} .
$$

- Pellegrino and Seoane-Sepúlveda ([4], 2012).

$$
\begin{aligned}
& \mathrm{B}_{\mathbb{K}, m}^{\text {mult }} \leq \mathrm{A}_{\mathbb{K}, \frac{2 m}{m+2}}^{-m / 2} \mathrm{~B}_{\mathbb{K}, m / 2}^{\text {mult }} \text { for } m \text { even, and } \\
& \mathrm{B}_{\mathbb{K}, m}^{\text {mult }} \leq\left(\mathrm{A}_{\mathbb{K}, \frac{2 m-2}{m+1}}^{\frac{-m-1}{\mathbb{K}, \frac{m-1}{2}}}\right)^{\frac{m-1}{2 m}}\left(\mathrm{~A}_{\mathbb{K}, \frac{2 m+2}{m+3}}^{\frac{1-m}{m u l t}} \mathrm{~B}_{\mathbb{K}, \frac{m+1}{2}}^{\text {mult }}\right)^{\frac{m+1}{2 m}} \text {, for } m \text { odd. }
\end{aligned}
$$

When $m$ is even and $k=m / 2$, we use Khinchine inequality to obtain estimates for the inequalities with the exponent

$$
\left(q_{1}, \ldots, q_{m}\right)=\left(\frac{2 m}{m+2}, \ldots, \frac{2 m}{m+2}, 2, \ldots, 2\right)
$$

and using the Minkowski inequality the same estimate is obtained for

$$
\left(q_{1}, \ldots, q_{m}\right)=\left(2, \ldots, 2, \frac{2 m}{m+2}, \ldots, \frac{2 m}{m+2}\right) .
$$


Using Proposition 5.2 we obtain

$$
\mathrm{B}_{\mathbb{K}, m}^{\mathrm{mult}} \leq \mathrm{A}_{\mathbb{K}, \frac{2 m}{m+2}}^{-m / 2} \mathrm{~B}_{\mathbb{K}, m / 2}^{\mathrm{mult}} .
$$

The case $m$ odd is somewhat similar, although it needs a little trick. It is worth mentioning that these estimates from [48] can be somewhat derived from abstract results appearing in [25].

5.2. Quantum Information Theory. Here we shall briefly describe a result by Montanaro [43, Theorem 5] which provided an application for the optimal Bohnenblust-Hille constants within the field of Quantum Physics. This presentation is based on Schwarting's Ph.D. dissertation [50, Section 2.2.5]. For a more detailed information we refer the interested reader to the Ph.D. dissertation of Briët [17, Chapter 1], which provides a very clear introduction to the whole topic of nonlocal games.

A classical nonlocal game is a pair $\mathcal{G}=(A, \pi)$ consisting on a function (called predicate) $A: \mathcal{A} \times \mathcal{B} \times \mathcal{S} \times \mathcal{S} \rightarrow\{ \pm 1\}$ and a probability distribution $\pi: \mathcal{S} \times \mathcal{T} \rightarrow[0,1]$. The game involves three parties: a person called the referee and two players (usually called Alice and Bob). When the game starts, the referee picks a question $(s, t) \in$ $\mathcal{S} \times \mathcal{T}$ according to the probability distribution $\pi$ and, then, sends it to Alice and Bob, who must reply independently (they are not allowed to communicate between each other once the game has begun) by providing an answer $a \in \mathcal{A}$ and $b \in \mathcal{B}$ each one. The players win the game if $A(a, b, s, t)=1$, and lose otherwise. The players' goal is to maximize their chance of winning. A XOR game is a nonlocal game in which the answer sets $\mathcal{A}, \mathcal{B}$ are $\{ \pm 1\}$ and the predicate $A$ depends only on the exclusive-OR (XOR) of the answers given by the players and the value of a Boolean function $\mathcal{S} \times \mathcal{T} \rightarrow\{ \pm 1\}$, which from the predicate may be seen as a matrix with entries on $\{ \pm 1\}$. A game with $m$-players is described similarly in the following fashion.

An $m$-player XOR (exclusive OR) game is a pair $\mathcal{G}=(\pi, A)$ consisting of a matrix $A=\left(a_{\mathbf{i}}\right)_{\mathbf{i} \in \mathcal{M}(m, n)}$, for which each entry $a_{\mathbf{i}} \in\{ \pm 1\}$, and a probability distribution $\pi: \mathcal{M}(m, n) \rightarrow[0,1]$. The game consists on the referee picking an $m$-tuple $\mathbf{i}=$ $\left(i_{1}, \ldots, i_{m}\right) \in \mathcal{M}(m, n)$ according to the probability distribution $\pi$ and sending each question $i_{k}$ to the player $k$, which, by means of a classical strategy, must reply upon this question with a (deterministic) answer map $y_{k}:\{1, \ldots, n\} \rightarrow\{ \pm 1\}$. The players win if and only if the product of their answers equals the corresponding entry in the matrix $A$, that is if

$$
y_{1}\left(i_{1}\right) \cdots y_{m}\left(i_{m}\right)=a_{\mathbf{i}}
$$

Concerning the complexity of a XOR game, one defines the bias $\beta(G)$ to be the greatest difference between the chance of winning and the chance of loosing the game for the optimal classical strategy. Therefore, the classical bias of an $m$-player $\mathrm{XOR}$ game is given by

$$
\beta(G)=\max _{y_{1}, \ldots, y_{m} \in\{ \pm 1\}^{n}}\left|\sum_{\mathbf{i} \in \mathcal{M}(m, n)} \pi(\mathbf{i}) a_{\mathbf{i}} y_{1}\left(i_{1}\right) \cdots y_{m}\left(i_{m}\right)\right| .
$$

If we define the $m$-linear map $T: \ell_{\infty}^{n} \times \cdots \times \ell_{\infty}^{n} \rightarrow \mathbb{R}$ by $T\left(e_{i_{1}}, \ldots, e_{i_{m}}\right):=a_{\mathbf{i}} \pi(\mathbf{i})$, then the bias will be

$$
\beta(G)=\|T\|
$$


A natural problem is to find the game for which the classical bias is minimized. It is known that there exists an $m$-player XOR game $\mathcal{G}$ for which

$$
\beta(\mathcal{G}) \leq n^{-\frac{m-1}{2}}
$$

(see 33]). Using the Bohnenblust-Hille inequality it is straightforward to obtain lower bounds for the classical bias of an $m$-player XOR games (see [43, Theorem $5])$.

Theorem 5.3. [43, Theorem 5] For every m-player XOR game $\mathcal{G}=(\pi, A)$,

$$
\beta(\mathcal{G}) \geq \frac{1}{\kappa m^{0.36482}} n^{\frac{1-m}{2}},
$$

where $\kappa>0$ is an universal constant.

Proof. Define the $m$-linear form $T: \ell_{\infty}^{n} \times \cdots \times \ell_{\infty}^{n} \rightarrow \mathbb{R}$ by $T\left(e_{i_{1}}, \ldots, e_{i_{m}}\right):=a_{\mathbf{i}} \pi(\mathbf{i})$. Then,

$$
\sum_{\mathbf{i} \in \mathcal{M}(m, n)}\left|T\left(e_{i_{1}}, \ldots, e_{i_{m}}\right)\right|=\sum_{\mathbf{i} \in \mathcal{M}(m, n)} \pi(\mathbf{i})=1 .
$$

Applying Hölder's inequality and the Bohnenblust-Hille, we conclude that

$$
\begin{aligned}
\sum_{\mathbf{i} \in \mathcal{M}(m, n)}\left|T\left(e_{i_{1}}, \ldots, e_{i_{m}}\right)\right| & \leq\left(\sum_{\mathbf{i} \in \mathcal{M}(m, n)}\left|T\left(e_{i_{1}}, \ldots, e_{i_{m}}\right)\right|^{\frac{2 m}{m+1}}\right)^{\frac{m+1}{2 m}}\left(\sum_{\mathbf{i} \in \mathcal{M}(m, n)} 1\right)^{\frac{m-1}{2 m}} \\
& \leq \mathrm{B}_{\mathbb{R}, m}^{\text {mult }} n^{\frac{m-1}{2}}\|T\|=\mathrm{B}_{\mathbb{R}, m}^{\text {mult }} n^{\frac{m-1}{2}} \beta(\mathcal{G}) .
\end{aligned}
$$

Using the best known estimates for the multilinear Bohnenblust-Hille inequality we conclude that

$$
\beta(\mathcal{G}) \geq \frac{1}{\kappa m^{\frac{2-\log 2-\gamma}{2}} n^{\frac{m-1}{2}}}>\frac{1}{\kappa m^{0.36482}} n^{\frac{1-m}{2}} .
$$

This result, according to Montanaro (see [43, p.4]), implies a very particular case of a conjecture of Aaronson and Ambainis (see [1]). Also, recent advances on the real polynomial Bohnenblust-Hille inequality (see, e.g., [18, 31]), combined with the CHSH inequality (due to Clauser, Horne, Shimony, and Holt in the late 1960 's), can be employed in the proof of Bell's theorem, which states that certain consequences of entanglement in quantum mechanics cannot be reproduced by local hidden variable theories. We refer the interested reader to the seminal paper, 21], in which more informtaion regarding this CHSH inequality can be found.

5.3. Power series and the Bohr radius problem . The following question was addressed by $\mathrm{H}$. Bohr in 1914:

How large can the sum of the mudulii of the terms of a convergent power series be?

The answer was given by the following theorem, which was independently obtain by Bohr, Riesz, Schur, and Wiener:

Theorem 5.4. Suppose that a power series $\sum_{k=0}^{\infty} c_{k} z^{k}$ converges for $z$ in the unit disk, and $\left|\sum_{k=0}^{\infty} c_{k} z^{k}\right|<1$ when $|z|<1$. Then $\sum_{k=0}^{\infty}\left|c_{k} z^{k}\right|<1$ when $|z|<1 / 3$. Moreover, the radius $1 / 3$ is the best possible. 
Following Boas and Khavinson [10, the Bohr radius $\mathrm{K}_{n}$ of the $n$-dimensional polydisk is the largest positive number $r$ such that all polynomials $\sum_{\alpha} a_{\alpha} z^{\alpha}$ on $\mathbb{C}^{n}$ satisfy

$$
\sup _{z \in r \mathbb{D}^{n}} \sum_{\alpha}\left|a_{\alpha} z^{\alpha}\right| \leq \sup _{z \in \mathbb{D}^{n}}\left|\sum_{\alpha} a_{\alpha} z^{\alpha}\right| .
$$

The Bohr radius $K_{1}$ was estimated by H. Bohr, M. Riesz, I. Schur and F. Wiener, and it was shown that $K_{1}=1 / 3$ (Theorem [5.4). For $n \geq 2$, exact values of $K_{n}$ are unknown. In [10, it was proved that

$$
\frac{1}{3} \sqrt{\frac{1}{n}} \leq \mathrm{K}_{n} \leq 2 \sqrt{\frac{\log n}{n}} .
$$

The paper by Boas and Khavinson, 10, motivated many other works, connecting the asymptotic behavior of $\mathrm{K}_{n}$ to various problems in Functional Analysis (geometry of Banach spaces, unconditional basis constant of spaces of polynomials, etc.); we refer to [26] for a panorama of the subject. Hence there was a big motivation in recent years in determining the behavior of $\mathrm{K}_{n}$ for large values of $n$.

In [23], the lefthand side inequality of (5.5) was improved to

$$
\mathrm{K}_{n} \geq c \sqrt{\log n /(n \log \log n)}
$$

In 24, using the hypercontractivity of the polynomial Bohnenblust-Hille inequality, the authors showed that

$$
\mathrm{K}_{n}=b_{n} \sqrt{\frac{\log n}{n}} \text { with } \frac{1}{\sqrt{2}}+o(1) \leq b_{n} \leq 2 .
$$

In this section we sketch how the Hölder inequality for mixed sums played a fundamental role in the final answer to the solution, given in [7], to the Bohr radius problem:

$$
\lim _{n \rightarrow \infty} \frac{\mathrm{K}_{n}}{\sqrt{\frac{\log n}{n}}}=1 .
$$

The solution has several ingredients, including the polynomial Bohnenblust-Hille inequality. Using (4.1), Bohnenblust and Hille were also able to have a polynomial version of this inequality: for any $m \geq 1$, there exists a constant $\mathrm{D}_{m} \geq 1$ such that, for any complex $m$-homogeneous polynomial $P(\mathbf{z})=\sum_{|\alpha|=m} a_{\alpha} \mathbf{z}^{\alpha}$ on $c_{0}$,

$$
\left(\sum_{|\alpha|=m}\left|a_{\alpha}\right|^{\frac{2 m}{m+1}}\right)^{\frac{m+1}{2 m}} \leq \mathrm{D}_{m}\|P\|,
$$

with

$$
\mathrm{D}_{m}=(\sqrt{2})^{m-1} \frac{m^{\frac{m}{2}}(m+1)^{\frac{m+1}{2}}}{2^{m}(m !)^{\frac{m+1}{2 m}}} .
$$

In fact, it is not difficult to use polarization and obtain the polynomial BohnenblustHille inequality by using the multilinear Bohnenblust-Hille inequality, but with bad constants (the following approach can be essentially found in [27, Lemma 5]). In 
fact, if $L$ is the polar of $P$, from (4.2) we have

$$
\begin{aligned}
\sum_{|\alpha|=m}\left|a_{\alpha}\right|^{\frac{2 m}{m+1}} & =\sum_{|\alpha|=m}\left(\left(\begin{array}{c}
m \\
\alpha
\end{array}\right)\left|L\left(e_{1}^{\alpha_{1}}, \ldots, e_{n}^{\alpha_{n}}\right)\right|\right)^{\frac{2 m}{m+1}} \\
& =\sum_{|\alpha|=m}\left(\begin{array}{c}
m \\
\alpha
\end{array}\right)^{\frac{2 m}{m+1}}\left|L\left(e_{1}^{\alpha_{1}}, \ldots, e_{n}^{\alpha_{n}}\right)\right|^{\frac{2 m}{m+1}} .
\end{aligned}
$$

However, for every choice of $\alpha$, the term

$$
\left|L\left(e_{1}^{\alpha_{1}}, \ldots, e_{n}^{\alpha_{n}}\right)\right|^{\frac{2 m}{m+1}}
$$

is repeated $\left(\begin{array}{c}m \\ \alpha\end{array}\right)$ times in the sum

$$
\sum_{i_{1}, \ldots, i_{m}=1}^{n}\left|L\left(e_{i_{1}}, \ldots, e_{i_{m}}\right)\right|^{\frac{2 m}{m+1}}
$$

Thus

$$
\sum_{|\alpha|=m}\left(\begin{array}{c}
m \\
\alpha
\end{array}\right)^{\frac{2 m}{m+1}}\left|L\left(e_{1}^{\alpha_{1}}, \ldots, e_{n}^{\alpha_{n}}\right)\right|^{\frac{2 m}{m+1}}=\sum_{i_{1}, \ldots, i_{m}=1}^{n}\left(\begin{array}{c}
m \\
\alpha
\end{array}\right)^{\frac{2 m}{m+1}} \frac{1}{\left(\begin{array}{c}
m \\
\alpha
\end{array}\right)}\left|L\left(e_{i_{1}}, \ldots, e_{i_{m}}\right)\right|^{\frac{2 m}{m+1}}
$$

and, since

$$
\left(\begin{array}{c}
m \\
\alpha
\end{array}\right) \leq m !
$$

we have

$$
\sum_{|\alpha|=m}\left(\begin{array}{c}
m \\
\alpha
\end{array}\right)^{\frac{2 m}{m+1}}\left|L\left(e_{1}^{\alpha_{1}}, \ldots, e_{n}^{\alpha_{n}}\right)\right|^{\frac{2 m}{m+1}} \leq(m !)^{\frac{m-1}{m+1}} \sum_{i_{1}, \ldots, i_{m}=1}^{n}\left|L\left(e_{i_{1}}, \ldots, e_{i_{m}}\right)\right|^{\frac{2 m}{m+1}} .
$$

We thus have

$$
\begin{aligned}
\left(\sum_{|\alpha|=m}\left|a_{\alpha}\right|^{\frac{2 m}{m+1}}\right)^{\frac{m+1}{2 m}} & \leq\left((m !)^{\frac{m-1}{m+1}} \sum_{i_{1}, \ldots, i_{m}=1}^{n}\left|L\left(e_{i_{1}}, \ldots, e_{i_{m}}\right)\right|^{\frac{2 m}{m+1}}\right)^{\frac{m+1}{2 m}} \\
& =(m !)^{\frac{m-1}{2 m}}\left(\sum_{i_{1}, \ldots, i_{m}=1}^{n}\left|L\left(e_{i_{1}}, \ldots, e_{i_{m}}\right)\right|^{\frac{2 m}{m+1}}\right)^{\frac{m+1}{2 m}} \\
& \leq(m !)^{\frac{m-1}{2 m}} \mathrm{~B}_{\mathbb{R}, m}^{\mathrm{mult}}\|L\| .
\end{aligned}
$$

On the other hand, since $\|L\| \leq \frac{m^{m}}{m !}\|P\|$ we obtain

$$
\begin{aligned}
\left(\sum_{|\alpha|=m}\left|a_{\alpha}\right|^{\frac{2 m}{m+1}}\right)^{\frac{m+1}{2 m}} & \leq \mathrm{B}_{\mathbb{R}, m}^{\text {mult }}(m !)^{\frac{m-1}{2 m}} \frac{m^{m}}{m !}\|P\| \\
& =\mathrm{B}_{\mathbb{R}, m}^{\text {mult }} \frac{m^{m}}{(m !)^{\frac{m+1}{2 m}}}\|P\| .
\end{aligned}
$$

Let us denote the best constant $\mathrm{D}_{m}$ in this inequality by $\mathrm{B}_{\mathbb{C}, m}^{\text {pol }}$. In 24 it was proved that in fact these estimates could be essentially improved to $(\sqrt{2})^{m-1}$. However using the variant of Hölder's inequality for mixed $\ell_{p}$ spaces, together with some results from Complex Analysis (see [7] for details) and with the subpolynomial 
estimates of the multilinear Bohnenblust-Hille inequality (Section 5), one of the main results of [7] shows that we can go much further:

Theorem 5.5. For any $\varepsilon>0$, there exists $\kappa>0$ such that, for any $m \geq 1$,

$$
\mathrm{B}_{\mathbb{C}, m}^{\mathrm{pol}} \leq \kappa(1+\varepsilon)^{m}
$$

As we mentioned above, in [24, using the hypercontractivity of the polynomial Bohnenblust-Hille inequality, the authors showed that

$$
\mathrm{K}_{n}=b_{n} \sqrt{\frac{\log n}{n}} \text { with } \frac{1}{\sqrt{2}}+o(1) \leq b_{n} \leq 2 .
$$

However, although (5.7) is quite precise, there was still uncertainity in the behavior of the number $b_{n}$. By combining classical tools of Complex Analysis (Harris' inequality [38]), Bayart's inequality [6], Wiener's inequality [7, Lemma 6.1], and the Kahane-Salem-Zygmund inequality (Theorem 4.5) together with Theorem 5.5 the authors, in [7, were finally able to provide the final solution to the Bohr radius problem:

Theorem 5.6. The asymptotic growth of the $n$-dimensional Bohr radius is $\sqrt{\frac{\log n}{n}}$. In other words, $\lim _{n \rightarrow \infty} \frac{\mathrm{K}_{n}}{\sqrt{\frac{\log n}{n}}}=1$.

The crucial step to complete the proof was the improvement of the estimates of the polynomial Bohnenblust-Hille inequality that was only achieved by means of the Hölder inequality for mixed sums.

5.4. Hardy-Littlewood's inequality constants. Although Hölder's inequality for mixed $\ell_{p}$ spaces dates back to the 1960's, its full importance in the subjects mentioned throughout this paper was just very recently realized. New consequences are still appearing (see, for instance [4,5, 19]). The last applications of the Hölder inequality for mixed $\ell_{p}$ spaces presented here concern the Hardy-Littlewood inequality and the theory of multiple summing multilinear operators. As in the case of the Bohnenblust-Hille inequality (Section 5) the Hölder inequality for multiple exponents allows a significant improvement in the constants of the Hardy-Littlewood inequality.

Let $\mathbb{K}$ be $\mathbb{R}$ or $\mathbb{C}$. Given an integer $m \geq 2$, the Hardy-Littlewood inequality (see [2, 37, 49]) asserts that for $2 m \leq p \leq \infty$ there exists a constant $\mathrm{C}_{m, p}^{\mathbb{K}} \geq 1$ such that, for all continuous $m$-linear forms $T: \ell_{p}^{n} \times \cdots \times \ell_{p}^{n} \rightarrow \mathbb{K}$ and all positive integers $n$,

$$
\left(\sum_{j_{1}, \ldots, j_{m}=1}^{n}\left|T\left(e_{j_{1}}, \ldots, e_{j_{m}}\right)\right|^{\frac{2 m p}{m p+p-2 m}}\right)^{\frac{m p+p-2 m}{2 m p}} \leq \mathrm{C}_{m, p}^{\mathbb{K}}\|T\| .
$$

Using the generalized Kahane-Salem-Zygmund inequality (see 2] ) one can easily verify that the exponents $\frac{2 m p}{m p+p-2 m}$ are optimal. When $p=\infty$, using that $\frac{2 m p}{m p+p-2 m}=\frac{2 m}{m+1}$, we recover the classical Bohnenblust-Hille inequality (see Theorem 5.2 and [12]).

From [7] we know that $\mathrm{B}_{\mathbb{K}, m}^{\text {mult }}$ has a subpolynomial growth. On the other hand, the best known upper bounds for the constants in (5.8) were, until just recently, 
$(\sqrt{2})^{m-1}$ (see [2, 3, 29]). Although, a suitable use of Theorem 3.2 shows that $(\sqrt{2})^{m-1}$ can be improved (see [5]) to

$$
\mathrm{C}_{m, p}^{\mathbb{R}} \leq(\sqrt{2})^{\frac{2 m(m-1)}{p}}\left(\mathrm{~B}_{\mathbb{R}, m}^{\text {mult }}\right)^{\frac{p-2 m}{p}}
$$

for real scalars and to

$$
\mathrm{C}_{m, p}^{\mathbb{C}} \leq\left(\frac{2}{\sqrt{\pi}}\right)^{\frac{2 m(m-1)}{p}}\left(\mathrm{~B}_{\mathbb{C}, m}^{\text {mult }}\right)^{\frac{p-2 m}{p}}
$$

for complex scalars. These estimates are substantially better than $(\sqrt{2})^{m-1}$ because $\mathrm{B}_{\mathbb{K}, m}^{\text {mult }}$ has a subpolynomial growth. In particular, if $p>m^{2}$ we conclude that $\mathrm{C}_{m, p}^{\mathbb{K}}$ has a subpolynomial growth.

5.5. Separately summing operators. Hölder's inequality is also used to generalize recent results on the theory of multiple summing multilinear operators. In 25], and for $m$-linear operators on $q$-cotype Banach spaces, the authors introduced the notion separately $(r, 1)$-summing, with $1 \leq r \leq q<\infty$, which means that, for any $(m-1)$-coordinates fixed, the resulting linear operator is $(r, 1)$-summing. Using separately summing maps, the authors concluded that the initial operator is multiple $\left(\frac{q r m}{q+(m-1) r}, 1\right)$-summing. In [4] it is presented the concept of $n$-separability summing, which stands for the $m$-linear operators that are multiple summing in $n$-coordinates, when there are $m-n$ other coordinates fixed. Using suitable interpolation, the authors provide $N$-separability from $n$-separability summing, with $n<N \leq m$. This result generalizes the previous one and provide more efficient exponents in some special cases. Moreover, it is also useful to provides estimates for the constants of some variation of Bohnenblust-Hille inequalities introduced in [46, Appendix A] and [47].

\section{REFERENCES}

[1] S. Aaronson and A. Ambainis, The need for structure in quantum speedups, In Proceedings of ICS (Innovations in Computer Science). Tsinghua University Press. arXiv:0911.0996. (2011), 338-352.

[2] N. Albuquerque, F. Bayart, D. Pellegrino, and J. B. Seoane-Sepúlveda, Sharp generalizations of the multilinear Bohnenblust-Hille inequality, J. Funct. Anal. 266 (2014), no. 6, 3726-3740, DOI 10.1016/j.jfa.2013.08.013.

[3] _ Optimal Hardy-Littlewood type inequalities for polynomials and multilinear operators, Israel J. Math., in press.

[4] N. Albuquerque, D. Núñez-Alarcón, J. Santos, and D. M. Serrano-Rodríguez, Absolutely summing multilinear operators via interpolation, arXiv:1404.4949 (2014).

[5] G. Araújo, D. Pellegrino, and D. D. P. da Silva e Silva, On the upper bounds for the constants of the Hardy-Littlewood inequality, J. Funct. Anal. 267 (2014), no. 6, 1878-1888, DOI 10.1016/j.jfa.2014.06.014.

[6] F. Bayart, Hardy spaces of Dirichlet series and their composition operators, Monatsh. Math. 136 (2002), 203-236.

[7] F. Bayart, D. Pellegrino, and J. B. Seoane-Sepúlveda, The Bohr radius of the ndimensional polydisk is equivalent to $\sqrt{(\log n) / n}$, Adv. Math. 264 (2014), 726-746, DOI http://dx.doi.org/10.1016/j.aim.2014.07.029.

[8] A. Benedek and R. Panzone, The space $L^{p}$, with mixed norm, Duke Math. J. 28 (1961), 301-324.

[9] J. Bergh and J. Löfström, Interpolation spaces. An introduction, Springer-Verlag, Berlin, 1976. Grundlehren der Mathematischen Wissenschaften, No. 223. 
[10] H. P. Boas and D. Khavinson, Bohr's power series theorem in several variables, Proc. Amer. Math. Soc. 125 (1997), no. 10, 2975-2979.

[11] H. P. Boas, Majorant series, J. Korean Math. Soc. 37 (2000), no. 2, 321-337. Several complex variables (Seoul, 1998).

[12] H. F. Bohnenblust and E. Hille, On the absolute convergence of Dirichlet series, Ann. of Math. (2) 32 (1931), no. 3, 600-622.

[13] H. Bohr, Über die gleichmäßige Konvergenz Dirichletscher Reihen, J. Reine Angew. Math. 143 (1913), 203-211.

[14] _ Lösung des absoluten Konvergenzproblems einer allgemeinen Klasse dirichletscher Reihen, Acta Math. 36 (1913), 197-240.

[15] — A theorem concerning power series, Proc. London Math. Soc. 13 (1914), 1-5.

[16] J. Bourgain, On the distribution of Dirichlet sums, J. Anal. Math. 60 (1993), 21-32, DOI 10.1007/BF03341964.

[17] J. Briët, Grothendieck Inequalities, Nonlocal Games and Optimization, 2011. Thesis (Ph.D.)Universiteit van Amsterdam.

[18] J. R. Campos, P. Jiménez-Rodríguez, G. A. Muñoz-Fernández, D. Pellegrino, and J. B. Seoane-Sepúlveda, On the real polynomial Bohnenblust-Hille inequality, Linear Algebra Appl., in press.

[19] D. Carando, A. Defant, and P. Sevilla-Peris, A note on Bohnenblust-Hille-Helson type inequality, arXiv:1403.7033 (2013).

[20] L. C. F. Ferreira, E. S. Medeiros, and M. Montenegro, A class of elliptic equations in anisotropic spaces, Ann. Mat. Pura Appl. (4) 192 (2013), no. 4, 539-552, DOI 10.1007/s10231-011-0236-8.

[21] J. Clauser, M. Horne, R. Shimony, and A. Holt, Proposed experiment to test local hiddenvariable theories, Phys. Rev. Lett. 23 (1969), no. 15, 880-4.

[22] A. M. Davie, Quotient algebras of uniform algebras, J. London Math. Soc. (2) 7 (1973), $31-40$.

[23] A. Defant and L. Frerick, A logarithmic lower bound for multi-dimensional Bohr radii, Israel J. Math. 152 (2006), 17-28.

[24] A. Defant, L. Frerick, J. Ortega-Cerdà, M. Ounaïes, and K. Seip, The Bohnenblust-Hille inequality for homogeneous polynomials is hypercontractive, Ann. of Math. (2) 174 (2011), no. 1, 485-497.

[25] A. Defant, D. Popa, and U. Schwarting, Coordinatewise multiple summing operators in Banach spaces, J. Funct. Anal. 259 (2010), no. 1, 220-242.

[26] A. Defant and C. Prengel, Harald Bohr meets Stefan Banach, Methods in Banach space theory, London Math. Soc. Lecture Note Ser., vol. 337, Cambridge Univ. Press, Cambridge, 2006, pp. 317-339.

[27] A. Defant and P. Sevilla-Peris, A new multilinear insight on Littlewood's 4/3-inequality, J. Funct. Anal. 256 (2009), no. 5, 1642-1664, DOI 10.1016/j.jfa.2008.07.005.

[28] A. Defant and P. Sevilla-Peris, The Bohnenblust-Hille cycle of ideas from a modern point of view, Funct. Approx. Comment. Math. 50 (2014), no. 1, [2013 on table of contents], 55-127, DOI 10.7169/facm/2014.50.1.2. MR3189502

[29] A. Dimant and P. Sevilla-Peris, Summation of coefficients of polynomials on $\ell_{p}$ spaces, arXiv:1309.6063v1 [math.FA].

[30] S. Dineen, Complex analysis on infinite-dimensional spaces, Springer Monographs in Mathematics, Springer-Verlag London, Ltd., London, 1999.

[31] D. Diniz, G. A. Muñoz-Fernández, D. Pellegrino, and J. B. Seoane-Sepúlveda, Lower bounds for the constants in the Bohnenblust-Hille inequality: the case of real scalars, Proc. Amer. Math. Soc. 142 (2014), 575-580.

[32] P. H. Enflo, V. I. Gurariy, and J. B. Seoane-Sepúlveda, On Montgomery's conjecture and the distribution of Dirichlet sums, J. Funct. Anal. 267 (2014), no. 4, 1241-1255, DOI 10.1016/j.jfa.2014.04.001.

[33] J. Ford and A. Gál, Hadamard tensors and lower bounds on multiparty communication complexity, Automata, languages and programming, Lecture Notes in Comput. Sci., vol. 3580, Springer, Berlin, 2005, pp. 1163-1175.

[34] J. J. F. Fournier, Mixed norms and rearrangements: Sobolev's inequality and Littlewood's inequality, Ann. Mat. Pura Appl. (4) 148 (1987), 51-76, DOI 10.1007/BF01774283. MR932758 (89e:46037) 
[35] D. J. H. Garling, Inequalities: a journey into linear analysis, Cambridge University Press, Cambridge, 2007.

[36] U. Haagerup, The best constants in the Khintchine inequality, Studia Math. 70 (1981), no. 3, 231-283 (1982).

[37] G. Hardy and J. E. Littlewood, Bilinear forms bounded in space [p,q], Quart. J. Math. 5 (1934), 241-254.

[38] L. A. Harris, Bounds on the derivatives of holomorphic functions of vectors, Analyse fonctionnelle et applications (Comptes Rendus Colloq. Analyse, Inst. Mat., Univ. Federal Rio de Janeiro, Rio de Janeiro, 1972), Hermann, Paris, 1975, pp. 145-163. Actualités Aci. Indust., No. 1367.

[39] O. Hölder, Über ein Mittelwertsatz, Nachr. Akad. Wiss. Göttingen Math. - Phys. Kl. (1889), $38-47$.

[40] J.-P. Kahane, Some random series of functions, 2nd ed., Cambridge Studies in Advanced Mathematics, vol. 5, Cambridge University Press, Cambridge, 1985.

[41] H. König, On the best constants in the Khintchine inequality for variables on spheres, Math. Seminar, Universität Kiel (1998).

[42] L. Maligranda, Why Hölder's inequality should be called Rogers' inequality, Math. Inequal. Appl. 1 (1998), no. 1, 69-83, DOI 10.7153/mia-01-05. MR1492911 (98j:01024)

[43] A. Montanaro, Some applications of hypercontractive inequalities in quantum information theory, J. Math. Physics 53 (2012), no. 12, 122206.

[44] H. L. Montgomery, Topics in multiplicative number theory, Lecture Notes in Mathematics, Vol. 227, Springer-Verlag, Berlin, 1971.

[45] J. Mujica, Complex analysis in Banach spaces, North-Holland Mathematics Studies, vol. 120, North-Holland Publishing Co., Amsterdam, 1986. Holomorphic functions and domains of holomorphy in finite and infinite dimensions; Notas de Matemática [Mathematical Notes], 107.

[46] D. Nuñez-Alarcón, D. Pellegrino, and J. B. Seoane-Sepúlveda, On the Bohnenblust-Hille inequality and a variant of Littlewood's 4/3 inequality, J. Funct. Anal. 264 (2013), no. 1, $326-336$.

[47] D. Nuñez-Alarcón, D. Pellegrino, J. B. Seoane-Sepúlveda, and D. M. Serrano-Rodríguez, There exist multilinear Bohnenblust-Hille constants $\left(C_{n}\right)_{n=1}^{\infty}$ with $\lim _{n \rightarrow \infty}\left(C_{n+1}-C_{n}\right)=0$, J. Funct. Anal. 264 (2013), no. 2, 429-463.

[48] D. Pellegrino and J. B. Seoane-Sepúlveda, New upper bounds for the constants in the Bohnenblust-Hille inequality, J. Math. Anal. Appl. 386 (2012), no. 1, 300-307.

[49] T. Praciano-Pereira, On bounded multilinear forms on a class of $l^{p}$ spaces, J. Math. Anal. Appl. 81 (1981), no. 2, 561-568, DOI 10.1016/0022-247X(81)90082-2.

[50] U. Schwarting, Vector Valued Bohnenblust-Hille Inequalities, 2013. Thesis (Ph.D.)Universität Oldenburg.

[51] A. Zygmund, Trigonometric series. Vol. I, II, 3rd ed., Cambridge Mathematical Library, Cambridge University Press, Cambridge, 2002.

Departamento de Matemática,, Universidade Federal da Paraíba,, 58.051-900 Jỗo PESSOA, BrazIL.

E-mail address: ngalbqrq@gmail.com

departamento de Matemática,, Universidade Federal da Paraíba,, $\quad$ 58.051-900 JoÃo Pessoa, BraziL.

E-mail address: gdasaraujo@gmail.com

Departamento de Matemática,, Universidade Federal da Paraíba,, 58.051-900 Jỗo Pessoa, BraziL.

E-mail address: dmpellegrino@gmail.com and pellegrino@pq.cnpq.br

Departamento de Análisis Matemático,, Facultad de Ciencias Matemáticas,, Plaza de Ciencias 3,, Universidad Complutense de Madrid,, Madrid, 28040, Spain., and, Instituto de Ciencias Matemáticas - ICMAT, Madrid, Spain.

E-mail address: jseoane@mat.ucm.es 